\section{Bone marrow from the iliac crest versus from the distal radius for revitalizing the necrotic lunate for Kienböck disease}

\section{Dear Editor,}

We developed a technically simple procedure to revitalize the necrotic lunate for Kienböck disease using a combination of non-concentrated bone marrow (BM) transfusion from the radius to lunate, lowintensity pulsed ultrasound, and external fixation
(Ogawa et al., 2013). The results were shown to be not inferior to those of conventional treatments for Kienböck disease (Ogawa et al., 2013). After 2007, our protocol was altered: non-concentrated BM from the iliac crest was used under the hypothesis that a greater number of BM cells would be advantageous to regenerate the necrotic lunate (Hernigou et al., 2005).

We treated 16 patients (nine men and seven women) with Kienböck disease using the iliac crest as the BM donor site. Preoperative Lichtman stages

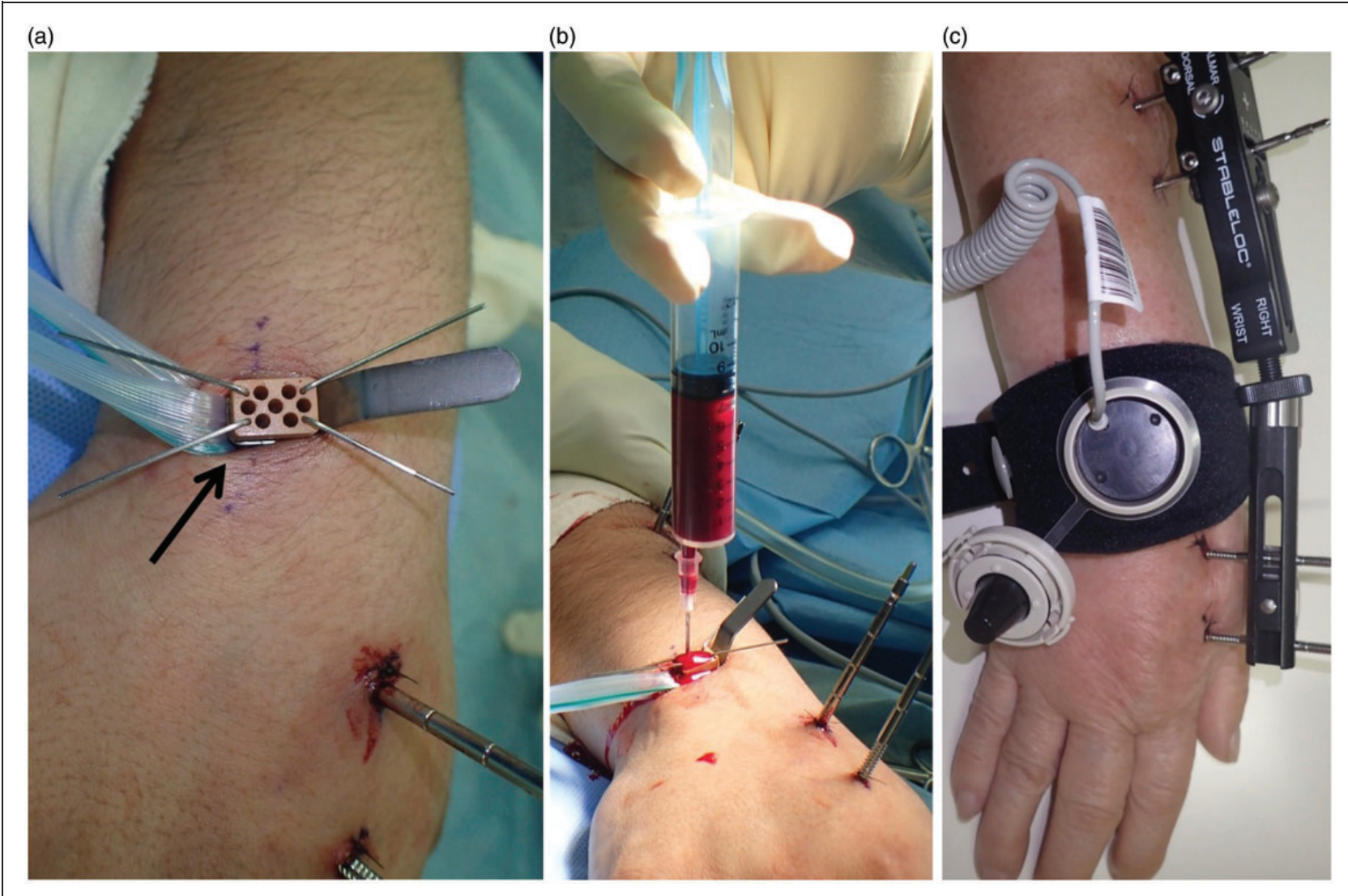

Figure 1. Our procedures. (a) A radiolucent drill guide (arrow) placed over the joint capsule while retracting the extensor tendon. (b) The transfusion method of the aspirated bone marrow from the iliac crest. (c) The external fixator is placed in slight traction. Ultrasound is applied on the dorsal wrist. 


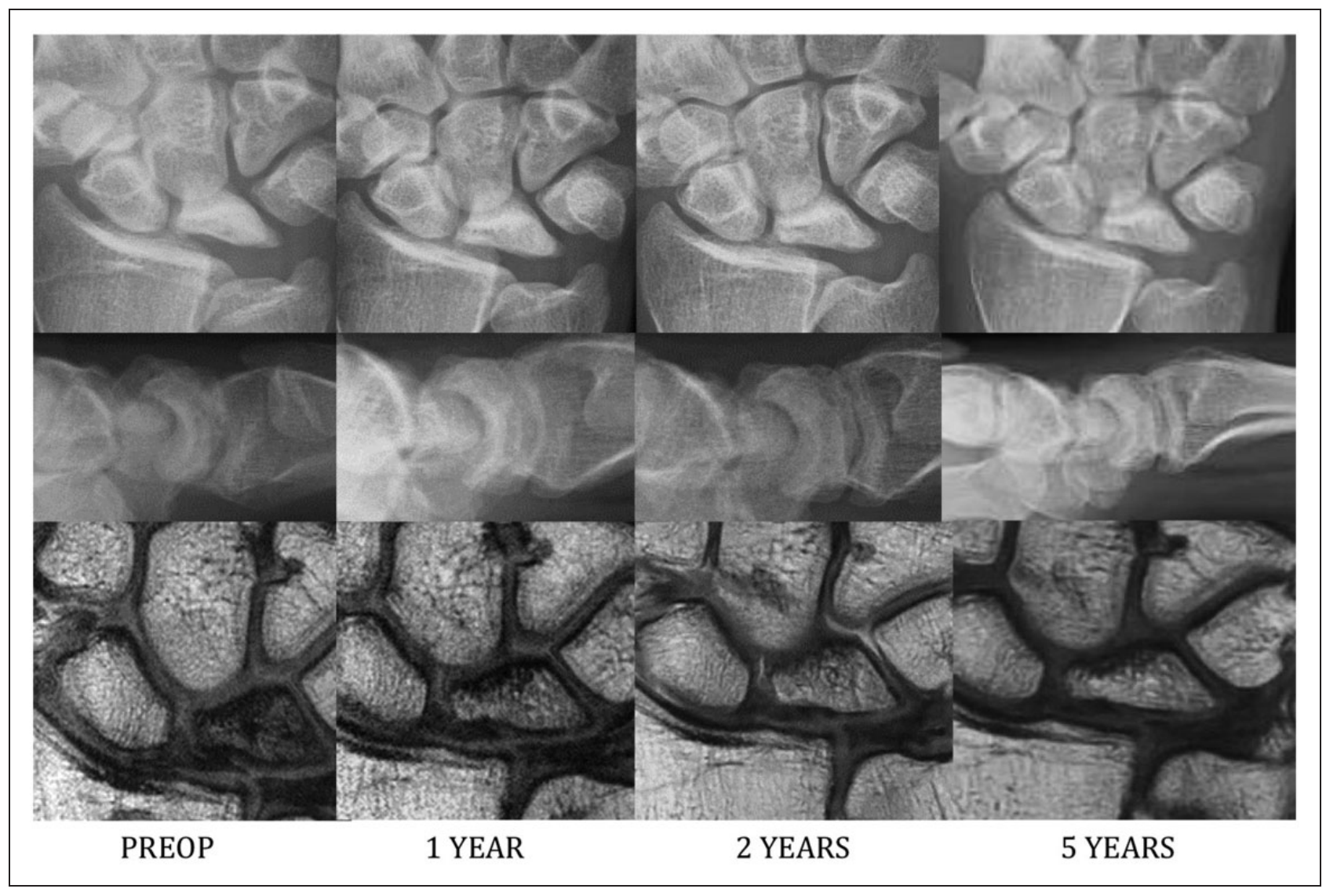

Figure 2. A 16-year-old man with Kienböck disease. On radiographs, the carpal height ratio and Stahl index increased slightly postoperatively. On MRI, the lunate intensity improved to almost normal over the first year.

were Stage II in five patients, Stage IIla in six, and Stage IIIb in five. We performed the surgery in the same way as in our previous report (Ogawa et al., 2013), except that we collected the BM aspirate (approximately $5 \mathrm{~mL}$ ) from the iliac crest (Figure 1). Mean age of the 16 patients at the surgery was 44 years (range 15-73); mean follow-up was 36 months (range 12-78). The length of external fixation was 69 days (range 49-90). The low-intensity pulsed ultrasound was used for 199 days (range 83-410).

The surgical outcomes were evaluated using the Mayo wrist score. The postoperative wrist motion ranges and strengths reported below are those from the final follow-up. We analysed the differences in the data obtained before the surgery and at the final follow-up after surgery using a paired $t$-test. Wrist pain decreased after surgery in all 16 patients, with no pain in 11 and mild pain in five at the final follow-up. The wrist flexion-extension significantly increased from $82^{\circ}$ (SD 31) before surgery to $107^{\circ}$ (SD 19) at final follow-up $(p=0.013)$. The grip strength increased from $42 \%$ (SD 17) before surgery to $82 \%$ (SD 11) at the follow-up relative to the unaffected side $(p<0.01)$. On radiographs, the carpal height ratio remained almost the same (from 0.516 to 0.518$)$, but the Stahl index significantly decreased from 0.37 (SD 0.07) to 0.32 (SD 0.1) ( $p=0.012)$. The Mayo wrist scores significantly improved from 47 (SD 7.7) preoperatively to 83 (SD 11) at follow-up $(p<0.01)$. The score was excellent in six, good in six, and satisfactory in four patients at the final follow-up. MRI revealed improvement in 13 patients (81\%) at follow-up (Figure 2). The improvement in the 16 patients was almost identical to that in the patients treated before 2007 and previously reported (Ogawa et al., 2013).

We used the iliac crest as BM with the hypothesis that a greater number of BM cells would better regenerate the necrotic lunate, but the clinical outcomes and MRI findings demonstrated almost the same results compared with our previous report (Ogawa et al., 2013). The presently reported series does again show the efficacy of the treatment with BM transfusion, ultrasound, and external fixation. The necessity of BM for the revitalizing necrotic bone was demonstrated by our clinical and basic study (Ogawa et al., 2010). Clinically, $2 \mathrm{~mL}$ of nonconcentrated BM is considered necessary and 
sufficient for necrotic lunate regeneration. We recommend using BM from the radius, namely our old protocol, because the BM can be easily collected from the same surgical area.

This study was not randomized, and the comparison is not strictly scientific. However, based on the 16 patients treated after 2007, our findings of almost identical outcomes prompted us to resume using the distal radius as the $\mathrm{BM}$ donor site.

Acknowledgements We thank Drs Yasumasa Nishiura and Masashi Yamazaki in Department of Orthopaedic Surgery at the University of Tsukuba for advice on clinical materials and basic science. We thank $\mathrm{Dr}$ Toshikazu Tanaka in Kikkoman General Hospital for leadership in surgeries. We thank Dr Yoshio Nakata of the Faculty of Health and Sport Sciences at the University of Tsukuba for advice on statistical analysis and Drs Sho Kohyama and Akira Ikumi for MRI examinations.

Declaration of conflicting interests The authors declare no potential conflicts of interest with respect to the research, authorship, and/or publication of this article.

Ethical approval The ethical committee of the University of Tsukuba approved our study (Number H27-139).

\section{References}

Hernigou P, Poignard A, Manicom O, Mathieu G, Rouard H. The use of percutaneous autologous bone marrow transplantation in nonunion and avascular necrosis of bone. J Bone Joint Surg Br. 2005, 87: 896-902.

Ogawa T, Ochiai N, Nishiura Y, Tanaka T, Hara Y. A new treatment strategy for Kienböck Disease: combination of bone marrow transfusion, low-intensity pulsed ultrasound therapy, and external fixation. J Orthop Sci. 2013, 18: 230-7.

Ogawa $T$, Ishii T, Mishima $H$ et al. Effectiveness of bone marrow transplantation for revitalizing a severely necrotic small bone: experimental rabbit model. J Orthop Sci. 2010, 15: $381-8$.

Takeshi Ogawa ${ }^{1,2, *}$, Naoyuki Ochiai ${ }^{3}$ and Yuki Hara ${ }^{2}$

${ }^{1}$ Department of Orthopaedic Surgery and Sports Medicine, Mito Clinical Education and Training Center, University of Tsukuba Hospital, Mito Kyodo General Hospital, Japan

${ }^{2}$ Department of Orthopaedic Surgery, Faculty of Medicine, University of Tsukuba, Japan

${ }^{3}$ Department of Orthopaedic Surgery, Kikkoman General Hospital, Japan

*Corresponding author: ogawatamd.tsukuba.ac.jp

(C) The Author(s) 2019

Article reuse guidelines:

sagepub.com/journals-permissions

doi: 10.1177/1753193419886724 available online at http://jhs.sagepub.com 